\title{
OSCILLATORY INTEGRALS AND FOURIER TRANSFORMS OF SURFACE CARRIED MEASURES
}

\author{
MICHAEL COWLING AND GIANCARLO MAUCERI
}

\begin{abstract}
We suppose that $S$ is a smooth hypersurface in $\mathbf{R}^{n+1}$ with Gaussian curvature $\kappa$ and surface measure $d S, w$ is a compactly supported cut-off function, and we let $\mu_{\alpha}$ be the surface measure with $d \mu_{\alpha}=w \kappa^{\alpha} d S$. In this paper we consider the case where $S$ is the graph of a suitably convex function, homogeneous of degree $d$, and estimate the Fourier transform $\hat{\mu}_{\alpha}$. We also show that if $S$ is convex, with no tangent lines of infinite order, then $\hat{\mu}_{\alpha}(\xi)$ decays as $|\xi|^{-n / 2}$ provided $\alpha \geq[(n+3) / 2]$. The techniques involved are the estimation of oscillatory integrals; we give applications involving maximal functions.
\end{abstract}

1. Introduction. The purpose of this paper is to obtain estimates for the decay at infinity of certain oscillatory integrals related to the Fourier transform of surface carried measures. Let $S$ denote a smooth hypersurface in $\mathbf{R}^{n+1}$ with Gaussian curvature $\kappa$ and element of surface measure $d S$ induced by the Lebesgue measure of $\mathbf{R}^{n+1}$. We fix a smooth function $w$ with compact support in $\mathbf{R}^{n+1}$ and a nonnegative number $\alpha$ and consider the finite Borel measure $\mu_{\alpha}$, with $d \mu_{\alpha}=$ $|\kappa|^{\alpha} w d S$, which is carried by $S$. We seek conditions on $S$ and $\alpha$ that guarantee that the Fourier transform $\left(\mu_{\alpha}\right)^{\wedge}$ of $\mu_{\alpha}$ satisfies the estimate

$$
\left|\left(\mu_{\alpha}\right)^{-}(\theta)\right| \leq c|\theta|^{-n / 2} \quad \forall \theta \in \mathbf{R}^{n+1} .
$$

This problem can be reduced to that of estimating the decay at infinity of an oscillatory integral. Indeed, by introducing a smooth partition of unity on $S$, we may assume that in a neighborhood of the support of $w$ the surface $S$ can be represented as the graph of a smooth function $f$ in $C_{c}^{\infty}\left(\mathbf{R}^{n}\right)$. Thus there exists a function $u$ in $C_{c}^{\infty}\left(\mathbf{R}^{n}\right)$ such that $d \mu_{\alpha}=\left|\operatorname{det} f^{\prime \prime}(x)\right|^{\alpha} u(x) d x$. Hence the Fourier transform of $\mu_{\alpha}$ can be written as an oscillatory integral

$$
I_{\alpha}(f, u)(\theta)=\int_{\mathbf{R}^{n}} \exp [i(\xi \cdot x+\lambda f(x))]\left|\operatorname{det}\left(f^{\prime \prime}(x)\right)\right|^{\alpha} u(x) d x,
$$

where $\theta=(\xi, \lambda)$ is a point in $\mathbf{R}^{n+1} \simeq \mathbf{R}^{n} \times \mathbf{R}$. The method of stationary phase (see, e.g., [H, Theorem 7.7.5]) shows that the decay given by the estimate

$$
\left|I_{\alpha}(f, u)(\theta)\right| \leq c|\theta|^{-n / 2} \quad \forall \theta \in \mathbf{R}^{n+1}
$$

is optimal, in the sense that for every nonlinear $f$ one can find a function $u$ in $C_{c}^{\infty}\left(\mathbf{R}^{n}\right)$ and a unit vector $\Theta$ in $\mathbf{R}^{n+1}$ such that $\left|I_{\alpha}(f, u)(\rho \Theta)\right| \sim C_{\alpha}(f, u) \rho^{-n / 2}$ as

Received by the editors October, $1,1986$.

1980 Mathematics Subject Classification (1985 Revision). Primary 42B10, 42B25.

The second author was supported in part by funds of the Ministero della Pubblica Istruzione. 
$\rho \rightarrow \infty$. Moreover if $\operatorname{det}\left(f^{\prime \prime}\right)$ never vanishes on the support of $u$, i.e. if the curvature of $S$ never vanishes on the support of $w,(1.2)$ holds.

Our interest in this problem arose in connection with the study of maximal averages of functions over hypersurfaces in $\mathbf{R}^{n+1}$. Define for every $t>0$ the "mean value" operator

$$
M_{t} \phi(x)=\int_{S} \phi(x-t y) w(y) d S(y) \quad \forall \phi \in S\left(\mathbf{R}^{n+1}\right)
$$

and the maximal operator

$$
\mathcal{M} \phi(x)=\sup \left\{\left|M_{t} \phi(x)\right|: t>0\right\} .
$$

One seeks conditions on the measure $\mu$ with $d \mu=w d S$ that guarantee that for some $p$ in $(1,+\infty)$ the estimate

$$
\|\mathcal{M} \phi\|_{p} \leq c\|\phi\|_{p} \quad \forall \phi \in S\left(\mathbf{R}^{n+1}\right)
$$

holds. A generalization of the methods introduced by E. M. Stein in his study of "the spherical maximal averages" $[\mathbf{S}, \mathbf{S W}]$ reduces the problem to that of obtaining decay estimates for the Fourier transform of the measure $\mu$ [CM, SS]. In particular if $\hat{\mu}$ vanishes at infinity of order less than $1 / 2$, one must obtain better decay for the Fourier transform of the measure $\mu_{\alpha}$, where $d \mu_{\alpha}=|\kappa|^{\alpha} d \mu$ for some positive $\alpha$. In [CM] the authors exhibited a class of surfaces in $\mathbf{R}^{3}$ for which $\left(\mu_{1 / 2}\right)^{\wedge}$ has optimal decay, i.e.

$$
\left|\left(\mu_{1 / 2}\right)^{\curlyvee}(\theta)\right| \leq c|\theta|^{-1} \quad \forall \theta \in \mathbf{R}^{3} .
$$

In [SS] Sogge and Stein proved that $\left(\mu_{2 n}\right)^{\wedge}$ has optimal decay for any smooth hypersurface $S$ in $\mathbf{R}^{n+1}$. In terms of oscillatory integrals this means that (1.2) is satisfied for every $u$ in $C_{c}^{\infty}\left(\mathbf{R}^{n}\right)$ if $f$ is smooth and $\alpha=2 n$.

Our goal is to improve this result for particular classes of functions $f$. In the next section we obtain decay estimates for the oscillatory integral $I$ :

$$
I(f, g, u)(\theta)=\int \exp [i(\xi \cdot x+\lambda f(x))] g(x) u(x) d x,
$$

where $\theta=(\xi, \lambda) \in \mathbf{R}^{n+1}$, and, roughly speaking, $f$ is a convex function homogeneous of degree $d \geq 2$, $\operatorname{det}\left(f^{\prime \prime}\right)$ vanishes only at the origin, $g$ is a homogeneous function of degree $z, \operatorname{Re} z+n>0$, and $u \in C_{c}^{\infty}\left(\mathbf{R}^{n}\right)$. These estimates show that, when $\alpha \geq 1 / 2, I_{\alpha}(f, u)=I\left(f, \operatorname{det}\left(f^{\prime \prime}\right)^{\alpha}, u\right)$ has the optimal decay in (1.2) for every $u$ in $C_{c}^{\infty}\left(\mathbf{R}^{n}\right)$. In $\S 3$ we apply these results to obtain sharp $L^{p}$-estimates for the maximal operator associated to a measure with compact support on the graph of $f$.

In $\S 4$ we derive decay estimates for a one-dimensional oscillatory integral whose phase function is convex, but no longer homogeneous. These results are applied in $\S 5$ to prove that if $S$ is a smooth convex hypersurface in $\mathbf{R}^{n+1}$ which has no tangent of infinite order than $\left(\mu_{\alpha}\right)^{-}$has optimal decay for $\alpha=[(n+3) / 2]$ (the integer part of $(n+3) / 2)$.

2. Oscillatory integrals with homogeneous phase. Let $f$ be a real-valued function, smooth on $\mathbf{R}^{n} \backslash\{0\}$, and homogeneous of degree $d \geq 2$. Assume further that for some positive constant $a$

$$
\left(f^{\prime \prime}(x) v, v\right) \geq a|v|^{2}
$$


for every $v, x \in \mathbf{R}^{n}$, with $|x|=1$. Then $f$ is convex and $\operatorname{det}\left(f^{\prime \prime}\right)$ vanishes only at the origin. Let $g$ be a smooth function on $\mathbf{R}^{n} \backslash\{0\}$, homogeneous of degree $z$. If $\operatorname{Re} z+n>0$ then $g$ is locally integrable. Thus for $u \in C_{c}^{\infty}\left(\mathbf{R}^{n}\right)$ and $\theta=(\xi, \lambda) \in$ $\mathbf{R}^{n} \times \mathbf{R}$ we may consider the oscillatory integral $I$ :

$$
I(f, g, u)(\theta)=\int_{\mathbf{R}^{n}} \exp [i(\xi \cdot x+\lambda f(x))] g(x) u(x) d x .
$$

Our goal in this section is to derive estimates for the decay of $I(f, g, u)(\theta)$ as $|\theta| \rightarrow \infty$. In particular we shall prove that if $\operatorname{Re} z+n \geq n d / 2$ then $I(f, g, u)$ has optimal decay.

The first step towards this result (which is Theorem 2.4 below) is Lemma 2.1 on the geometry of the graph of $f$. This is followed by Proposition 2.2 on the Fourier transform of the function $x \rightarrow \exp [i f(x)] g(x)$. Lemma 2.3 is a technical result used for proving Proposition 2.2, and the main result, Theorem 2.4, then follows.

LEMMA 2.1. For any $\xi$ in $\mathbf{R}^{n}$ there exists a unique $x(\xi)$ in $\mathbf{R}^{n}$ such that $f^{\prime}(x(\xi))=-\xi$. Moreover there exist constants $b, c, c(d)>0$ such that

(i) $b|\xi|^{1 /(d-1)} \leq|x(\xi)| \leq c|\xi|^{1 /(d-1)}$,

(ii) $\left|\xi+f^{\prime}(x)\right| \geq c(d) a|x(\xi)|^{d-2}|x-x(\xi)|$,

(iii) $\left|\xi+f^{\prime}(x)\right| \geq c(d) a|x|^{d-2}|x-x(\xi)|$, for every $x$ in $\mathbf{R}^{n}$.

Proof. Since $f$ is strictly convex and $f(x) \geq a d^{-1}(d-1)^{-1}|x|^{d}$ by (2.1), the set $\Sigma=\{x: f(x)=1\}$ is the boundary of a strictly convex body. For every $x$ in $\Sigma$ let $\mathbf{n}(x)$ denote the exterior normal to $\Sigma$ at $x$. Thus for every unit vector $\sigma$ in $\mathbf{R}^{n}$ there exists $x_{\sigma}$ in $\Sigma$ such that

$$
\mathbf{n}\left(x_{\sigma}\right)=f^{\prime}\left(x_{\sigma}\right)\left|f^{\prime}\left(x_{\sigma}\right)\right|^{-1}=\sigma .
$$

For $\xi$ in $\mathbf{R}^{n} \backslash\{0\}$ set $\sigma=-\xi /|\xi|$ and $x(\xi)=\left(|\xi| /\left|f^{\prime}\left(x_{\sigma}\right)\right|\right)^{1 /(d-1)} x_{\sigma}$. Then it is easily seen that $f^{\prime}(x(\xi))=-\xi$ and $|x(\xi)| \sim|\xi|^{1 /(d-1)}$. This proves (i). The uniqueness of $x(\xi)$ will follow from estimate (ii). To prove the estimate write, for the sake of brevity, $\phi(x)=\xi \cdot x+f(x)$. Then

$$
\begin{aligned}
\phi^{\prime}(x)(x-x(\xi)) & =\int_{0}^{1} f^{\prime \prime}(x(\xi)+t(x-x(\xi)))(x-x(\xi), x-\{(\xi)) d t \\
& \geq a|x-x(\xi)|^{2} \int_{0}^{1}|x(\xi)+t(x-x(\xi))|^{d-2} d t
\end{aligned}
$$

by (2.1) and the homogeneity of $f$. Since it is easily see that

$$
|x(\xi)+t(x-x(\xi))|=|x(\xi)|\left|1-\left\{|x(\xi)|^{-1}|x-x(\xi)|\right\} t\right|,
$$

we have

$$
\left|\phi^{\prime}(x)\right| \geq a|x(\xi)|^{d-2}|x-x(\xi)| \int_{0}^{1}\left|1-\left\{|x(\xi)|^{-1}|x-x(\xi)|\right\} t\right|^{d-2} d t .
$$

Since there exists a constant $c(d)>0$ such that

$$
\int_{0}^{1}|1-\alpha t|^{d-2} d t \geq c(d) \quad \forall \alpha \geq 0
$$


(ii) follows from (2.2). Interchanging the roles of $x$ and $x(\xi)$, we can use the same argument to prove (iii).

Let $T_{f, g}(x)=e^{i f(x)} g(x)$. Since $\operatorname{Re} z+n>0, T_{f, g}$ defines a tempered distribution on $\mathbf{R}^{n}$.

PROPOSITION 2.2. If $\operatorname{Re} z+n>0$ then $\hat{T}_{f, g} \in C^{\infty}\left(\mathbf{R}^{n}\right)$. If further $0<$ $\operatorname{Re} z+n \leq n d / 2$, then there exists a constant $c(f, g)$ such that, for every $\xi$ in $\mathbf{R}^{n}$,

$$
\left|\hat{T}_{f, g}(\xi)\right| \leq c(f, g) .
$$

PROOF. The basic idea of the proof consists in expressing the Fourier transform of the distribution $T_{f, g}$ as an oscillatory integral with phase function $\phi(x, \xi)=$ $\xi \cdot x+f(x)$. The function $x \rightarrow \phi(x, \xi)$ is in $C^{2}\left(\mathbf{R}^{n}\right)$, is smooth away from the origin, and for every $\xi \neq 0$ has a unique nondegenerate stationary point $x(\xi)$. Then we decompose the domain of integration into three regions: a neighborhood of the stationary point that does not contain the origin, a neighborhood of the origin that does not contain the stationary point, and a neighborhood of infinity. In the first region the required estimate will follow by the stationary phase argument, while in the last two regions it will follow by integration by parts.

If $m \in \mathbf{R}$ we denote by $S^{m}\left(\mathbf{R}^{n}\right)$ the symbol class of all functions $a$ in $C^{\infty}\left(\mathbf{R}^{n}\right)$ such that for every multi-index $\alpha$ in $\mathbf{N}^{n}$ there exists a constant $c(\alpha)$ such that

$$
\left|\partial^{\alpha} a(x)\right| \leq c(\alpha)(1+|x|)^{m-|\alpha|} \quad \forall x \in \mathbf{R}^{n} .
$$

We shall prove first that if $\operatorname{Re} z+n>0$ then $\hat{T}_{f, g} \in C^{\infty}\left(\mathbf{R}^{n}\right)$. Let $\zeta$ be a function in $C_{c}^{\infty}\left(\mathbf{R}^{n}\right)$ such that $0 \leq \varsigma(x) \leq 1, \varsigma(x)=1$ if $|x| \leq 1 / 2$, and $\varsigma(x)=0$ if $|x| \geq 2$. Then, if $s_{t}(x)=\varsigma(t x)$,

$$
\hat{T}_{f, g}(\xi)=\lim _{t \rightarrow 0+} \int e^{i \phi(x, \xi)} g(x) \zeta_{t}(x) d x
$$

in $S^{\prime}\left(\mathbf{R}^{n}\right)$. By (i) and (iii) of Lemma 2.1, for every positive $R$ there exist positive constants $c_{2}$ and $c(f, R)$ such that

$$
\left|\phi_{x}^{\prime}(x, \xi)\right| \geq c_{2}(1+|x|)^{d-1}
$$

if $|\xi| \leq R$ and $|x| \geq c(f, R)$. Now let $\psi$ be a function in $C_{c}^{\infty}\left(\mathbf{R}^{n}\right)$ such that $0 \leq \psi(x) \leq 1, \psi(x)=1$ for $|x| \leq c(f, R)$, and $\psi(x)=0$ for $|x| \geq 2 c(f, R)$. Set $\chi=1-\psi$. Then

$$
\begin{aligned}
\hat{T}_{f, g}(\xi)= & \int_{\mathbf{R}^{n}} e^{i \phi(x, \xi)} g(x) \psi(x) d x \\
& +\lim _{t \rightarrow 0+} \int_{\mathbf{R}^{n}} e^{i \phi(x, \xi)} g(x) \chi(x) \zeta_{t}(x) d x .
\end{aligned}
$$

The first integral in (2.5) is an entire function of $\xi$. To handle the second integral consider the differential operator

$$
L u(x)=i \sum_{j=1}^{n} \partial_{j}\left(\left|\phi_{x}^{\prime}(x, \xi)\right|^{-2} \partial_{j} \phi(x, \xi) u(x)\right),
$$

where the derivatives $\partial_{j}$ are taken with respect to the variable $x$. The operator $L$ maps functions supported away from the origin in the symbol class $S^{m}\left(\mathbf{R}^{n}\right)$ into 
the symbol class $S^{m-d}\left(\mathbf{R}^{n}\right)$. Moreover its formal transpose $L^{t}$ satisfies $L^{t} e^{i \phi}=e^{i \phi}$. Since the function $x \rightarrow g(x) \chi(x)_{\zeta}(t x)$ is supported away from the origin and is in the symbol class $S^{\operatorname{Re} z}\left(\mathbf{R}^{n}\right)$ uniformly with respect to $t$ in [0,1], by integrating by parts $k$ times we get

$$
\int_{\mathbf{R}^{n}} e^{i \phi(x, \xi)} g(x) \chi(x) \zeta(t x) d x=\int_{\mathbf{R}^{n}} e^{i \phi(x, \xi)} L^{k}\left(g \chi \zeta_{t}\right)(x) d x,
$$

where $L^{k}\left(g \chi \varsigma_{t}\right) \in S^{\operatorname{Re} z-d}\left(\mathbf{R}^{n}\right)$, uniformly with respect to $t$ in $[0,1]$. Thus, if $k>\operatorname{Re} z+n$, then by the Lebesgue dominated convergence theorem

$$
\lim _{t \rightarrow 0+} \int e^{i \phi(x, \xi)} g \chi \zeta_{t}(x) d x=\int e^{i \phi(x, \xi)} g \chi(x) d x .
$$

The latter integral converges absolutely and defines a smooth function of $\xi$. Consequently

$$
\hat{T}_{f, g}(\xi)=\int_{\mathbf{R}^{n}} e^{i \phi(x, \xi)}\left[g \psi+L^{k} g \chi\right](x) d x,
$$

$\hat{T}_{f, g}(\xi)$ is a smooth function of $\xi$, and

$$
\left|\hat{T}_{f, g}(\xi)\right| \leq c(g) \quad \forall|\xi| \leq 1 .
$$

Then to obtain estimate (2.3) we only need to investigate the behavior of $\hat{T}_{f, g}(\xi)$ as $|\xi| \rightarrow \infty$. Letting $\omega=\xi /|\xi|$ and performing the change of variables $x=|\xi|^{1 /(d-1)} y$ in (2.4), we get

$$
\begin{aligned}
& |\xi|^{-(z+n) /(d-1)} \hat{T}_{f, g}(\xi) \\
& \quad=\lim _{t \rightarrow 0+} \int_{\mathbf{R}^{n}} \exp \left[i|\xi|^{d /(d-1)}(\omega \cdot y+f(y))\right] g(y) \zeta_{t}(y) d y .
\end{aligned}
$$

Estimate (2.3) is then an immediate consequence of the following lemma.

Lemma 2.3. Let $r>0, \omega \in \mathbf{R}^{n},|\omega|=1$. Define

$$
I_{\omega}(r)=\lim _{t \rightarrow 0+} \int_{\mathbf{R}^{n}} \exp [i r(\omega \cdot y+f(y))] g(y) \zeta_{t}(y) d y .
$$

Then there exists a constant $c_{3}$, independent of $\omega$, such that

$$
\left|I_{\omega}(r)\right| \leq c_{3}(1+r)^{-\min \{\operatorname{Re} z+n, n / 2\}} \quad \forall r \in \mathbf{R}^{+} .
$$

PROOF. Let $\phi(y, \omega)=\omega \cdot y+f(y), \omega, y$ in $\mathbf{R}^{n},|\omega|=1$. The function $y \rightarrow \phi(y, \omega)$ is in $C^{\infty}\left(\mathbf{R}^{n} \backslash\{0\}\right) \cup C^{2}\left(\mathbf{R}^{n}\right)$ and, by Lemma 2.1 , has a unique critical point $y(\omega)$ for every unit vector $\omega$ in $\mathbf{R}^{n}$. Moreover there exist positive $\omega$-independent constants $c_{4}, c_{5}, \varepsilon_{0}, \varepsilon_{1}$, such that

(i) $\varepsilon_{0} \leq|y(\omega)| \leq \varepsilon_{1}$,

(ii) $\left|\phi_{y}^{\prime}(y, \omega)\right| \geq c_{4}(1+|y|)^{d-1}$ if $|y-y(\omega)| \geq 1$,

(iii) $\operatorname{det}\left(\phi_{y y}^{\prime \prime}(y(\omega), \omega)\right) \geq c_{5}$,

(iv) $\left|\partial_{y}^{\alpha} \phi(y, \omega)\right| \leq c(\alpha)|y|^{d-|\alpha|} \forall y \in \mathbf{R}^{n} \backslash\{0\}, \forall \alpha \in \mathbf{N}^{n},|\alpha| \geq 2$.

Since these estimates hold uniformly with respect to $\omega,|\omega|=1$, we shall forget altogether the dependence on $\omega$ and write $\phi(y)$ instead of $\phi(y, \omega)$ and $y_{0}$ instead of $y(\omega)$. Now let $\psi_{1}, \psi_{2}$, and $\psi_{3}$ be $C^{\infty}\left(\mathbf{R}^{n}\right)$-functions such that

(a) $0 \leq \psi_{i} \leq 1, \sum_{i=1}^{3} \psi_{i}=1$, 
(b) $\psi_{1}$ has support in a ball $B_{1}$ of center $y_{0}$ which does not contain the origin,

(c) $\psi_{2}$ has support in a small ball $B_{2}$ of center the origin, to be chosen later, which does not contain $y_{0}$.

Using this partition of unity we can write

$$
\int_{\mathbf{R}^{n}} e^{i r \phi(y)} g(y) \zeta_{t}(y) d y=\sum_{j=1}^{3} I_{j}(t, r),
$$

where $I_{j}(t, r), j=1,2$, is the integral over $B_{j}$. Now

$$
\lim _{t \rightarrow 0} I_{1}(t, r)=I_{1}(r)=\int_{\mathbf{R}^{n}} e^{i r \phi(y)} g(y) \psi_{1}(y) d y
$$

this is an oscillatory integral, with a smooth phase function which has a unique nondegenerate stationary point in the support of the amplitude. Thus by the stationary phase theorem $\left[\mathbf{H}\right.$, Theorem 7.7.5] there exists a constant $c_{6}$ such that

$$
\left|I_{1}(r)\right| \leq c_{6}(1+r)^{-m / 2} \quad \forall r>0 .
$$

Next

$$
\lim _{t \rightarrow 0+} I_{2}(t, r)=I_{2}(r)=\int_{\mathbf{R}^{n}} e^{i r \phi(y)} g(y) \psi_{2}(y) d y .
$$

Integrating by parts $k$ times, where $k$ is the largest integer less than $\operatorname{Re} z+n$, we get

$$
I_{2}(r)=r^{-k} \int_{\mathbf{R}^{n}} e^{i r \phi(y)} L^{k}\left(g \psi_{2}\right)(y) d y
$$

where

$$
L f(y)=i \sum_{j=1}^{n} \partial_{j}\left[\left|\phi^{\prime}(y)\right|^{-2} \partial_{j} \phi(y) f(y)\right] .
$$

An easy induction argument shows that $L^{k}\left(g \psi_{2}\right)$ is a sum of terms $\left(L^{j} g\right)\left(L^{k-j} \psi_{2}\right)$; in this product, one factor is homogeneous and the other is smooth and compactly supported. Therefore the function $a_{g}=L^{k}\left(g \psi_{2}\right)$ is in the Besov space $\Lambda_{\operatorname{Re} z-k+n}^{1, \infty}\left(\mathbf{R}^{n}\right)$. Moreover if we choose $B_{2}$ sufficiently small there exists a $C^{2}$ diffeomorphism $\Phi$ of a neighborhood of the origin onto $B_{2}$ and a unit vector $v$ in $\mathbf{R}^{n}$ such that $\phi \circ \Phi(x)=v \cdot x$. Thus $I_{2}(r)=r^{-k}\left[\left(a_{g} \circ \Phi\right) \mid \Phi^{\prime} \|\right]^{\wedge}(r v)$ and so

$$
\left|I_{2}(r)\right| \leq c_{7} r^{-(\operatorname{Re} z+n)} \text {. }
$$

Finally to estimate $I_{3}(t, r)$ we integrate by parts $j$ times, obtaining

$$
I_{3}(t, r)=r^{-j} \int e^{i r \phi(y)} L^{j} a_{g}(y, t, r) d y,
$$

where $a_{g}(y, t, r)=g(y) \varsigma\left(\operatorname{tr}^{\delta} y\right) \psi_{3}(y)$ is a symbol of class $S^{\operatorname{Re} z}\left(\mathbf{R}^{n}\right)$, uniformly with respect to $t, r>0$. Thus since $L$ maps symbols in $S^{m}\left(\mathbf{R}^{n}\right)$ supported away from the origin into $S^{m-d}\left(\mathbf{R}^{n}\right)$, we have for all $j$ large enough

$$
I_{3}(r)=\lim _{t \rightarrow 0+} I_{3}(t, r)=r^{-j} \int e^{i r \phi(y)} L^{j}\left(g \psi_{3}\right)(y) d y .
$$

This shows that $I_{3}(r)$ is a rapidly decreasing function of $r$ as $r \rightarrow \infty$, and completes the proof of Lemma 2.3 . 
THEOREM 2.4. If $0<\operatorname{Re} z+n$ then there exists a constant $c=c(n, f, g, u)$ such that

$$
|I(f, g, u)(\theta)| \leq c|\theta|^{-\min \{n / 2,(\operatorname{Re} z+n) / d\}} \quad \forall \theta \in \mathbf{R}^{n+1} .
$$

In particular if $\operatorname{Re} z+n \geq n d / 2$ then

$$
|I(f, g, u)(\theta)| \leq c|\theta|^{-n / 2} \quad \forall \theta \in \mathbf{R}^{n+1} .
$$

Since for $\alpha$ real, $\operatorname{det}\left(f^{\prime \prime}\right)^{\alpha}$ is homogeneous of degree $\alpha n(d-2)$, the following corollary is an immediate consequence of Theorem 2.1.

COROLLARY 2.5. If $\alpha \geq 1 / 2$ then there exists a constant $c=c(n, \alpha, f, u)$ such that, when $\theta=(\xi, \lambda)$,

$$
\left|\int_{\mathbf{R}^{n}} \exp [i(\xi \cdot x+\lambda f(x))] \operatorname{det}\left(f^{\prime \prime}(x)\right)^{\alpha} u(x) d x\right| \leq c|\theta|^{-n / 2} .
$$

Proof of Theorem 2.4. Now if $t>0$ and $x \in \mathbf{R}^{n}$ let $\delta_{t} x=t x$. Since $g$ is homogeneous of degree $z$ we have for every $\lambda>0$

$$
e^{i \lambda f(x)} g(x)=\lambda^{-z / d}\left[T_{f, g} \delta_{\lambda^{1 / d}}\right](x) .
$$

Hence, for $\theta=(\xi, \lambda)$ in $\mathbf{R}^{n} \times \mathbf{R}$ and $u$ in $C_{c}^{\infty}\left(\mathbf{R}^{n}\right)$,

$$
\begin{aligned}
I(f, g, u)(\theta) & =\lambda^{-z / d}\left[\left(T_{f, g} \delta_{\lambda^{1 / d}}\right) u\right]^{-}(\xi) \\
& =\lambda^{-(z+n) / d}\left[\left(\hat{T}_{f, g} \delta_{\lambda^{-1 / d}}\right) * \hat{u}\right](\xi) .
\end{aligned}
$$

Therefore, if $0<\operatorname{Re} z+n \leq n d / 2$, we have by Lemma 2.3 ,

$$
\begin{aligned}
|I(f, g, u)(\theta)| & \leq \lambda^{-(\operatorname{Re} z+n) / d}\left\|\hat{T}_{f, g}\right\|_{\infty}\|\hat{u}\|_{1} \\
& \leq c(f, g)\|\hat{u}\|_{1} \lambda .
\end{aligned}
$$

If $\operatorname{Re} z+n>n d / 2$, by the same argument we get

$$
|I(f, g, u)(\theta)| \leq c(f, g)\left\|\left(|x|^{\varepsilon} u\right)^{-}\right\|_{1} \lambda^{-n / 2},
$$

where $\varepsilon=\operatorname{Re} z+n-n d / 2>0$. Notice that

$$
\left\|\left(|x|^{\varepsilon} u\right)^{\wedge}\right\|_{1}=\left\|\left(|x|^{\varepsilon}\right)^{\wedge} * \hat{u}\right\|_{1}<\infty,
$$

since $\hat{u} \in S\left(\mathbf{R}^{n}\right)$ and $\left(|x|^{\varepsilon}\right)^{\wedge}$ is a homogeneous distribution of degree $-n-\varepsilon$. Thus combining (2.7) and (2.8) we get

$$
|I(f, g, u)(\theta)| \leq c(f, g, u, \delta)|\theta|^{-\min \{n / 2,(\operatorname{Re} z+n) / d\}},
$$

in any cone $\Gamma(\delta)=\{(\xi, \lambda):|\xi| \leq \delta \lambda\}$. If $\lambda<0$ the same estimate follows by considering the conjugate of $I(f, g, u)(\theta)$. To estimate the oscillatory integral in the complement of one of the cones $\Gamma(\delta)$ we use Proposition 2.2. Let

$$
h(x)=h(x ; \xi, \lambda)=|\xi|^{-1}(\xi \cdot x+\lambda f(x)) .
$$

We claim that there exist constants $\delta_{0}, e>0$ such that

$$
\inf \left\{\left|h^{\prime}(x)\right|: x \in \operatorname{supp}(u)\right\} \geq e \quad \forall|\xi| \geq \delta_{0}|\lambda| .
$$

Indeed $h^{\prime}(x)=|\xi|^{-1}\left(\xi+\lambda f^{\prime}(x)\right)$ and by Lemma 2.1, there exists a unique $x(\xi / \lambda)$ such that $h^{\prime}(x(\xi / \lambda))=0$, unless $\lambda=0$ in which case (2.9) is trivial. Moreover 
$|x(\xi / \lambda)| \sim|\xi / \lambda|^{-1 /(d-1)}$. Thus there exists $\delta_{0}>0$ such that for $|\xi| \geq \delta_{0}|\lambda|$ we have $\operatorname{dist}(x(\xi / \lambda), \operatorname{supp}(u)) \geq 1$. Finally, by (iii) of Lemma 2.1, if $x \in \operatorname{supp}(u)$,

$$
\begin{aligned}
\left|h^{\prime}(x)\right| & \geq c_{1}|\lambda||\xi|^{-1}|\xi / \lambda|^{(d-2) /(d-1)}|x-x(\xi / \lambda)| \\
& \geq c_{1}|\xi / \lambda|^{-1 /(d-1)} \max \{1,|x(\xi / \lambda)|-|x|\} \\
& \geq e>0 .
\end{aligned}
$$

Thus we can integrate by parts $k$ times in the integral $I(f, g, u)(\theta)$, where $k$ is the largest integer less than $\operatorname{Re} z+n$, obtaining

$$
\begin{aligned}
I(f, g, u)(\theta) & =\int_{\mathbf{R}^{n}} e^{i|\xi| h(x)} g(x) u(x) d x \\
& =|\xi|^{-k} \int_{\mathbf{R}^{n}} e^{i|\xi| h(x)} L^{k}(g u)(x) d x,
\end{aligned}
$$

where $L$ is the different operator $i \sum_{j=1}^{n} \partial_{j}\left[|\nabla h|^{-2} \partial_{j} h\right]$ whose formal transpose $L^{t}$ satisfies $L^{t} e^{i|\xi| h}=|\xi| e^{i|\xi| h}$. Now an induction argument shows that $a_{g}=L^{k}(g u)$ is a sum $\sum_{p} a_{p} b_{p}$, where each $a_{p}$ is a function in $C^{\infty}\left(\mathbf{R}^{n} \backslash\{0\}\right)$ which is homogeneous of degree $\lambda, \operatorname{Re} \lambda \geq \operatorname{Re} \dot{z}-k$, and $b_{p} \in C_{c}^{2}\left(\mathbf{R}^{n}\right)$. Thus $a_{g}$ is in the Besov space $\Lambda_{\operatorname{Re} z-k+n}^{1, \infty}\left(\mathbf{R}^{n}\right)$. Now, since $\left|h^{\prime}\right|$ is bounded away from zero on the support of $u$, we can decompose $u$ with a partition of unity into a sum $u=\sum_{j} u_{j}$ of smooth functons $u_{j}$ with compact support in balls $B_{j}$, in such a way that $h$ can be taken as a coordinate in a new local coordinate system in a neighborhood of each $B_{j}$. More precisely, for every $j$ there exists a unit vector $\omega_{j}$ in $\mathbf{R}^{n}$ and a $C^{2}$ diffeomorphism $\Phi_{j}$ of a neighborhood of the origin onto $B_{j}$ such that $h \circ \Phi_{j}(x)=x \cdot \omega_{j}$. Thus

$$
I(f, g, u)(\theta)=|\xi|^{-k} \sum_{j}\left[\left(a_{g} \circ \Phi_{j}\right)\left|\Phi_{j}^{\prime}\right|^{\wedge}\left(|\xi| \omega_{j}\right)\right.
$$

and, since $\left(a_{g} \circ \Phi_{j}\right)\left|\Phi_{j}^{\prime}\right| \in \Lambda_{\operatorname{Re} z-k+n}^{1, \infty}\left(\mathbf{R}^{n}\right)$, we have

$$
\begin{aligned}
|I(f, g, u)(\theta)| & \leq \sum_{j}\left\|\left(a_{g} \circ \Phi_{j}\right)\left|\Phi_{j}^{\prime}\right|\right\|_{\Lambda_{\operatorname{Re} z-k+n}^{1, \infty}}|\xi|^{-(\operatorname{Re} z+n)} \\
& \leq c(n, f, g, u)|\xi|^{-(\operatorname{Re} z+n)}
\end{aligned}
$$

for $|\xi|>\delta_{0}|\lambda|$. This proves the theorem.

3. Estimates for maximal functions. In this section we shall apply the results of the previous section to derive $L^{p}$ estimates, $1<p \leq \infty$, for maximal operators associated to averages over a hypersurface $S$ in $\mathbf{R}^{n+1}$ which, modulo a rotation and a translation, is the graph of a homogeneous function $f: \mathbf{R}^{n} \rightarrow \mathbf{R}$. We shall assume that $f$ satisfies the assumptions of $\S 2$, to wit $f$ is a smooth function in $\mathbf{R}^{n} \backslash\{0\}$, homogeneous of degree $d \geq 2$, and there exists a positive constant $a$ such that $\left(f^{\prime \prime}(x) v, v\right) \geq a|v|^{2}$ for every $x, v$ in $\mathbf{R}^{n},|x|=1$. Let $\mu$ be a finite Borel measure of the form $d \mu=w d S$, where $d S$ is the surface measure on $S$ and $w$ is a function with compact support on $S$ such that the function

$$
x \rightarrow u(x)=w(x, f(x))\left(1+\left|f^{\prime}(x)\right|^{2}\right)^{-1 / 2}
$$

is in $C_{c}^{\infty}\left(\mathbf{R}^{n}\right)$. We shall prove that the maximal operator

$$
\mathcal{M} \phi(x)=\sup _{t>0}\left|\int_{S} \phi(x-t y) d \mu(y)\right| \quad \forall \phi \in S\left(\mathbf{R}^{n+1}\right)
$$


is bounded on $L^{p}\left(\mathbf{R}^{n+1}\right)$ for every $p>p(n, d)$. The critical exponent $p(n, d)$ is less than 2 or larger than 2 according as $d<2 n$ or $d \geq 2 n$.

THEOREM 3.1. Suppose $n \geq 2$. If $d<2 n$ then $\mathcal{M}$ is bounded on $L^{p}\left(\mathbf{R}^{n+1}\right)$ for every $p>1+(d / 2 n)$. If $d \geq 2 n$ then $\mathcal{M}$ is bounded on $L^{p}\left(\mathbf{R}^{n+1}\right)$ for every $p>d / n$.

ProOF. First, suppose that $d<2 n$. By [CM, Theorem 2.2] it suffices to show

$$
|\hat{\mu}(\theta)| \leq c(1+|\theta|)^{-n / d} \quad \forall \theta \in \mathbf{R}^{n+1} .
$$

([CM] treats surfaces which are starlike relative to the origin. The same method, with "Riesz operators" centered at a point $P$, establishes the analogous result for surfaces which are starlike relative to $P$.)

Since $|\hat{\mu}(\theta)|=|I(f, 2, u)(\theta)|$, estimate (3.1) follows at once from Theorem 2.1.

Next assume that $d \geq 2 n$ and denote by $\kappa$ the Gaussian curvature of $S$. If $\alpha>-(d-2)^{-1}$ then $\kappa^{\alpha}$ is integrable relative to surface measure. Let $d \mu_{\alpha}=\kappa^{\alpha} d \mu$. By Theorem 2.1 if $\alpha>(d-2 n) / 2 n(d-2)$ there exists $\varepsilon>0$ such that

$$
\left|\left(\mu_{\alpha}\right)^{-}(\theta)\right|=\left|I\left(f, \operatorname{det}\left(f^{\prime \prime}\right)^{\alpha}, u\right)\right| \leq c(1+|\theta|)^{-1 / 2-\varepsilon} \quad \forall \theta \in \mathbf{R}^{n+1} .
$$

The conclusion now follows from [CM, Theorem 3.2].

REMARK. If $d \geq 2 n$ the result of Theorem 3.1 is sharp. Indeed let $S$ be the hypersurface $x_{n+1}=f\left(x^{\prime}\right)-1, x^{\prime} \in \mathbf{R}^{n}$, and let $u$ in $C_{c}^{\infty}\left(\mathbf{R}^{n}\right)$ be a function such that $u\left(x^{\prime}\right)=1$ in a neighborhood of the origin. Consider the function $\phi(x)=$ $\eta(x)\left|x_{n+1}\right|^{-1 / p}\left(\log 2\left|x_{n+1}\right|\right)^{-1}$, where $\eta$ is a nonnegative $C_{c}^{\infty}$ function supported in the unit ball, with $\eta=1$ near the origin. Then $\phi$ is in $L^{p}\left(\mathbf{R}^{n+1}\right)$. However for $p \leq d / n$ the average

$$
M_{t} \phi\left(x^{\prime}, x_{n+1}\right)=\int_{\mathbf{R}^{n}} \phi\left(x^{\prime}-t y^{\prime}, x_{n+1}-t\left(f\left(y^{\prime}\right)-1\right)\right) u\left(y^{\prime}\right) d y^{\prime}
$$

is infinite for $x_{n+1}<0$ and $t=\left|x_{n+1}\right|$. This is precisely the value of $t$ that brings together in convolution the singularity of $\phi$ and the point where the curvature of $S$ vanishes. Thus

$$
\mathcal{M} \phi\left(x^{\prime}, x_{n+1}\right)=+\infty \quad \forall x_{n+1}<0 .
$$

4. A one-dimensional oscillatory integral. Let $I$ be the interval $[0,1]$. For every $m \in \mathbf{N}$ and every function $f \in C^{m}(I)$ we shall denote by $\|f\|_{(m)}$ the norm of $f$ in $C^{m}(I)$ :

$$
\|f\|_{(m)}=\max \left\{\left|f^{(i)}(t)\right|: t \in I, 0 \leq i \leq m\right\} .
$$

In this section we shall consider functions $\phi \in C^{p+1}(I), \psi \in C^{p-1}(I)$, and $u \in$ $C^{p}(I), p \geq 3$, such that

$\phi$ is a convex function on $I, \phi(0)=\phi^{\prime}(0)=0$, and there exist an integer $q$ in $[2, p]$ and a positive constant $\varepsilon$ such that

$$
\max \left\{\left|\phi^{(i)}(0)\right|: 2 \leq i \leq q\right\} \geq \varepsilon
$$

(4.2) there exists a positive constant $c_{0}$ such that $0 \leq \psi(t) \leq c_{0} \phi^{\prime \prime}(t)$ for $t \in I$;

$$
\text { there exists a constant } M \text { such that }
$$

$$
\|\phi\|_{(p+1)} \leq M \quad \text { and }\|\psi\|_{(p-1)} \leq M
$$




$$
u(t)=1 \text { if } 0 \leq t \leq 1 / 3 \text { and } u(t)=0 \text { if } 2 / 3 \leq t \leq 1 .
$$

Our goal is to give estimates for the oscillatory integral $I_{k}$ :

$$
I_{k}(\lambda)=\int_{0}^{1} \exp [i \lambda \phi(t)] \psi(t)^{k+1} u(t) t^{2 k-1} d t \quad \forall \lambda \in \mathbf{R}
$$

for $k$ a positive integer. We begin by proving an easy result on $\phi^{\prime}$ (Lemma 4.1), and then we estimate certain derivatives of $t \rightarrow \psi(t)^{k+1} t^{2 k-1}$, first in an easy case (Lemma 4.2) then in a more difficult situation (Lemma 4.3). These estimates enable us to prove our main estimate (Proposition 4.4) by integration by parts.

LEMMA 4.1. If $\phi$ satisfies hypotheses (4.1) and (4.3) then there exists a positive constant $c(\varepsilon, p, M)$ such that

$$
\phi^{\prime}(t) \geq c(\varepsilon, p, M) \quad \forall t \in[1 / 3,1] .
$$

ProOF. The set $\mathbf{F}$ of all $\phi$ in $C^{p+1}(I)$ which satisfy (4.1) and (4.3) is compact in $C^{p}(I)$, and $\phi \rightarrow \phi^{\prime}(1 / 3)$ is a continuous functional on $C^{p}(I)$; consequently, there exists $\phi_{0}$ in $\mathbf{F}$ so that, for any $\phi$ in $E$, and $t$ in $[1 / 3,1]$,

$$
\phi_{0}^{\prime}(1 / 3) \leq \phi^{\prime}(1 / 3) \leq \phi^{\prime}(t) .
$$

Since $\phi(0)=\phi_{0}^{\prime}(0)=0$ and $\phi_{0}^{\prime}$ is increasing in $I$, if $\phi_{0}^{\prime}(1 / 3)$ were 0 , then $\phi_{0}$ would be identically zero in $[0,1 / 3]$, contradicting $(4.1)$. So $\phi_{0}^{\prime}(1 / 3)>0$, as required.

(See also Lemma 3.3 and the following remark in $[\mathbf{S v}]$.

Let $D_{\phi}$ be the differential operator given by the rule

$$
D_{\phi} f(t)=\frac{d}{d t}\left[\phi^{\prime}(t)^{-1} f(t)\right] \quad \forall f \in C^{1}(I)
$$

and write $j$ for the identity function: $j(t)=t, t \in I$.

LEMMA 4.2. Suppose $\phi$ and $\psi$ satisfy (4.1), (4.2), and (4.3). Assume that for some integer $m \in[1, q-1]$ one has

$$
\phi^{(i)}(0)=0, \quad i=0, \ldots, m,\left|\phi^{(m+1)}(0)\right| \geq \varepsilon .
$$

Then there exists a constant $c=c\left(p, q, \varepsilon, c_{0}, M, k\right)$ such that

$$
\left\|D_{\phi}^{k}\left(\psi^{k+1} j^{2 k-1}\right)\right\|_{\infty} \leq c .
$$

PROOF. Since $\phi$ is convex, $\phi^{(m+1)}(0) \geq \varepsilon$. A simple induction argument on $k$ shows that

$$
D_{\phi}^{k}\left(\psi^{k+1} j^{2 k-1}\right)(t)=\sum_{P} P\left(t, D_{t}, \psi(t), F(t), G(t)\right)
$$

where $D_{t}=d / d t, F(t)=t \psi(t) / \phi^{\prime}(t), G(t)=t^{2} \psi^{\prime}(t) / \phi^{\prime}(t)$, and the sum runs over a finite set of polynomials $P$ in the variables $t, D_{t}, \psi, F$, and $G$ of degree at most $k$ in $D_{t}$. Thus we only need to prove that the norms of $\psi, F$, and $G$ in $C^{k}(I)$ are bounded by a constant depending on $p, q, \varepsilon, c_{0}$, and $M$. By (4.3) this is obvious for $\psi$, since $p \geq k+1$. To estimate the norms of $F$ and $G$ we write

$$
F(t)=\left[t^{m} / \phi^{\prime}(t)\right]\left[\psi(t) / t^{m-1}\right] \text { and } G(t)=\left[t^{m} / \phi^{\prime}(t)\right]\left[\psi^{\prime}(t) / t^{m-2}\right] .
$$

By Taylor's formula with integral remainder,

$$
\psi(t)=t^{m-1} \xi(t) \quad \text { and } \quad \psi^{\prime}(t)=t^{m-2} \eta(t)
$$


where $\xi \in C^{p-m}(I)$ while $\eta \in C^{p-m}(I)$ if $m \geq 2$ and $\eta \in C^{p-2}(I)$ if $m=1$. At any rate, since $k \leq \min \{p-m, p-2\}, \xi$ and $\eta$ are both in $C^{k}(I)$ and

$$
\|\xi\|_{(k)}+\|\eta\|_{(k)} \leq c(p, q)\|\psi\|_{(p-1)} \leq c(p, q, M) .
$$

Hence we only need to estimate the norm of $j^{m} / \phi^{\prime}$ in $C^{k}(I)$. Consider first the function $\phi^{\prime} / j^{m}$. Again by Taylor's formula we have $\phi^{\prime}(t)=t^{m} \alpha(t)$, where $\alpha \in$ $C^{k}(I)$ and

$$
\|\alpha\|_{(k)} \leq c(p, q)\|\phi\|_{(p+1)} \leq c(p, q, M) .
$$

Next we claim that there exists a positive constant $c(\varepsilon, M)$ such that

$$
|\alpha(t)| \geq c(\varepsilon, M) \quad \forall t \in I .
$$

Indeed, since $\phi^{(m+1)}(0) \geq \varepsilon$, we have

$$
\phi^{m+1}(t) \geq \varepsilon / 2 \quad \forall t \in\left[0, \varepsilon / 2\|\phi\|_{(p+1)}\right] .
$$

Thus for $0 \leq t \leq \varepsilon / 2\|\phi\|_{(p+1)}$,

$$
\alpha(t)=\phi^{\prime}(t) t^{-m}=[(m-1) !]^{-1} \int_{0}^{1}(1-u)^{m-1} \phi^{(m+1)}(t u) d u \geq \varepsilon / 2[m] ! .
$$

On the other hand, since $\phi^{\prime}$ is increasing, we have for $\varepsilon / 2\|\phi\|_{(p+1)} \leq t \leq 1$

$$
\begin{aligned}
\alpha(t) & \geq \phi^{\prime}\left(\varepsilon / 2\|\phi\|_{(p+1)}\right) t^{-m} \\
& \geq\left(\varepsilon / 2\|\phi\|_{(p+1)}\right)^{m} \varepsilon[m !] \geq c(\varepsilon, q, M)>0 .
\end{aligned}
$$

Thus $j^{m} / \phi^{\prime}=\alpha^{-1}$ is in $C^{k}(I)$ and

$$
\left\|j^{m} / \phi^{\prime}\right\|_{(k)} \leq c(p, q, \varepsilon, M) .
$$

This proves the lemma.

LEMMA 4.3. Let $\phi$ and $\psi$ satisfy hypotheses (4.1)-(4.3). If

$$
p \geq \max (k+q-1, k+2)
$$

then the function $D_{\phi}^{k}\left(\psi^{k+1} j^{2 k-1}\right)$ is bounded in $I$ and there exists a constant $B=$ $B\left(p, \varepsilon, c_{0}, M, k\right)$ such that

$$
\left\|D_{\phi}^{k}\left(\psi^{k+1} j^{2 k-1}\right)\right\|_{\infty} \leq B .
$$

If $l<k$ then we have also

$$
\lim _{t \rightarrow 0+} D_{\phi}^{l}\left(\psi^{k+1} j^{2 k-1}\right)(t)=0 .
$$

ProOF. To prove (4.6) just consider the Taylor expansions of $j^{2 k-1} \psi^{k+1}$ and $\phi^{\prime}$ centered at 0 . We shall prove estimate (4.5) by induction on $m$ :

$$
m=\min \left\{n \in \mathbf{N}:\left|\phi^{(n)}(0)\right| \geq \varepsilon\right\} .
$$

At most $q-2$ steps will be required since we start when $m=2$ and stop when $m=q$. If $m=2$, Lemma 4.2 applies. Next suppose that estimate (4.5) holds whenever $2 \leq m \leq \mu$ and take $\phi$ such that

$$
\min \left\{n \in \mathbf{N}:\left|\phi^{(n)}(0)\right| \geq \varepsilon\right\}=\mu+1 .
$$


If $\phi^{(n)}(0)=0$ for $n=0, \ldots, \mu$ we are done by Lemma 4.2 . It remains to treat the case where there is some $n \in[2, \mu]$ with $\phi^{(n)}(0) \neq 0$. For $s, t$ in $I$, let

$$
\phi_{s}(t)=s^{-1-\mu} \phi(s t), \quad \psi_{s}(t)=s^{1-\mu} \psi(s t) .
$$

Since

$$
\begin{aligned}
& \phi_{s}^{(i)}(t)=s^{i-1-\mu} \phi^{(i)}(s t), \quad i=0, \ldots, p+1, \\
& \psi_{s}^{(i)}(t)=s^{i+1-\mu} \psi^{(i)}(s t), \quad i=0, \ldots, p-1,
\end{aligned}
$$

we have

$$
0 \leq \psi_{s}(t) \leq c_{0} \phi_{s}^{\prime \prime}(t) \quad \forall t \in I,
$$

and $\phi_{s}^{(i)}(0)=s^{i-1-\mu} \phi^{(i)}(0)$. Since, for $0 \leq i \leq \mu,\left|\phi_{s}^{(i)}(0)\right|$ is a decreasing function of $s$ which tends to $\infty$ as $s \rightarrow 0+$, there exists a critical value $\sigma$ in $(0,1)$ such that

$$
\max \left\{\left|\phi_{s}^{(i)}(0)\right|: 0 \leq i \leq \mu\right\} \gtreqless \varepsilon \text { as } s \lesseqgtr \sigma .
$$

Moreover there exists a constant $\bar{M}=\bar{M}(q, \varepsilon, M)$ such that

$$
\left\|\phi_{s}\right\|_{(p+1)}+\left\|\psi_{s}\right\|_{(p-1)} \leq \bar{M} \quad \forall s \in(0,1) .
$$

Indeed to prove (4.11) observe that by (4.7) if $i \geq \mu+1$,

$$
\left|\phi_{s}^{(i)}(t)\right| \leq\left\|\phi^{(i)}\right\|_{\infty} \leq M \quad \forall t \in I .
$$

If $i \leq \mu$ we have by (4.10)

$$
\left|\phi_{s}^{(i)}(t)\right| \leq\left|\phi_{s}^{(i)}(0)\right|+\int_{0}^{t}\left|\phi_{s}^{(i+1)}(u)\right| d u \leq \varepsilon+\left\|\phi_{s}^{(i+1)}\right\|_{\infty} .
$$

Hence, by a backward induction argument on $i$ :

$$
\left\|\phi_{s}^{(i)}\right\|_{\infty} \leq(\mu+1-i) \varepsilon+\left\|\phi^{(\mu+1)}\right\|_{\infty}, \quad i=0, \ldots, \mu .
$$

This proves that

$$
\left\|\phi_{s}\right\|_{(p+1)} \leq(\mu+1) \varepsilon+\|\phi\|_{(p+1)} \leq(q+1) \varepsilon+M .
$$

Finally, to prove that $\left\|\psi_{s}\right\|_{(p-1)}$ is bounded uniformly with respect to $s$, we use (4.8), (4.9), and the well-known estimates

$$
\left\|\psi_{s}^{(i)}\right\|_{\infty} \leq \gamma\left(\left\|\psi_{s}\right\|_{\infty}+\left\|\psi_{s}^{(p-1)}\right\|_{\infty}\right), \quad i=0, \ldots, p-1 .
$$

Next it is easily seen that for $t, s$ in $I$,

$$
D_{\phi_{s}}^{k}\left(\psi_{s}^{2 k-1} j^{2 k-1}\right)(t)=s^{2-k-\mu}\left[D_{\phi}^{k}\left(\psi^{k+1} j^{2 k-1}\right)\right](s t) .
$$

Since $k+\mu-2 \geq 1$ we have

$$
\left|D_{\phi}^{k}\left(\psi^{k+1} j^{2 k-1}\right)(s t)\right| \leq\left|D_{\phi_{s}}^{k}\left(\psi_{s}^{k+1} j^{2 k-1}\right)(t)\right| .
$$

If $0 \leq s \leq \sigma$ we can find $t$ in $[0,1]$ such that $s=\sigma t$. Since $\phi_{\sigma}$ and $\psi_{\sigma}$ satisfy (4.1)-(4.3) with $q=\mu$ and $\bar{M}$ instead of $M$, we get by (4.12) and the inductive hypothesis

$$
\left|D_{\phi}^{k}\left(\psi^{k+1} j^{2 k-1}\right)(s)\right| \leq\left|\left(D_{\phi_{\sigma}}\right)^{k}\left(\psi_{\sigma}^{k+1} j^{2 k-1}\right)(t)\right| \leq B\left(p, \varepsilon, c_{0}, \bar{M}, k\right) .
$$


To estimate $\left|D_{\phi}^{k}\left(\psi^{k+1} j^{2 k-1}\right)(s)\right|$ when $\sigma \leq s \leq 1$, we observe that (4.12) yields

$$
\left|D_{\phi}^{k}\left(\psi^{k+1} j^{2 k-1}\right)(s)\right| \leq\left|D_{\phi_{s}}^{k}\left(\psi_{s}^{k+1} j^{2 k-1}\right)(1)\right| .
$$

The functions $\phi_{s}$ and $\psi_{s}$ for $\sigma \leq s \leq 1$ satisfy conditions (4.2)-(4.3), with $M$ replaced by $\bar{M}$. Let $\mathbf{K}$ be the set of all pairs of functions $(\phi, \psi)$ in $C^{p+1}(I) \times$ $C^{p-1}(I)$ satisfying (4.2)-(4.3) for a fixed choice of the constants $q, \varepsilon, c_{0}, M$. By the compactness of the injection $C^{m+1}(I) \rightarrow C^{m}(I), \mathbf{K}$ is relatively compact in $C^{p}(I) \times C^{p-2}(I)$. Moreover by Lemma 4.1, $\phi^{\prime}(1)$ is bounded below for $(\phi, \psi)$ in K. Thus there exists a constant $c(\mathbf{K})$ such that $\left|D_{\phi}^{k}\left(\psi^{k+1} j^{2 k-1}\right)(1)\right| \leq c(\mathbf{K})$ for all $(\phi, \psi)$ in $\mathbf{K}$. This proves the lemma.

PROPOSITION 4.4. Let $\phi, \psi$, and $u$ satisfy hypotheses (4.1)-(4.4). If $p \geq$ $\max (k+q-1, k+2)$ then there exists a constant $a=a\left(p, \varepsilon, c_{0}, M, u, k\right)$ such that

$$
\left|I_{k}(\lambda)\right| \leq a|\lambda|^{-k} \quad \forall \lambda \in \mathbf{R} .
$$

ProOF. We can estimate $I_{k}(\lambda)$ by integrating by parts $k$ times; thus

$$
I_{k}(\lambda)=(-i \lambda)^{-k} \int_{0}^{1} \exp [i \lambda \phi(t)] D_{\phi}^{k}\left(\psi^{k+1} j^{2 k-1} u\right)(t) d t
$$

and so

$$
\left|I_{k}(\lambda)\right| \leq\left\|D_{\phi}^{k}\left(\psi^{k+1} j^{2 \kappa-1} u\right)\right\|_{\infty}|\lambda|^{-k} \quad \forall \lambda \in \mathbf{R} .
$$

By expanding out the derivatives, we find that

$$
D_{\phi}^{k}\left(\psi^{k+1} j^{2 k-1} u\right)=u D_{\phi}^{k}\left(\psi^{k+1} j^{2 k-1}\right)+\text { other terms. }
$$

Each of the other terms involves a derivative of $u$ and so it vanishes outside $[1 / 3,2 / 3]$. By Lemma 4.1 and Leibnitz' rule the contribution of the other terms is bounded by a constant which depends only on $\varepsilon, c_{0}, M, u$, and $k$. The estimate for $D_{\phi}^{k}\left(\psi^{k+1} j^{2 k-1}\right)$ is the content of Lemma 4.3 .

5. Estimates for convex surfaces. Let $S$ denote a smooth convex hypersurface in $\mathbf{R}^{n+1}$ (not necessarily closed), with Gaussian curvature $\kappa$ and induced Lebesgue measure $d S$. Let $w \in C_{c}^{\infty}(S)$ be a function compactly supported away from the boundary of $S$.

THEOREM 5.1. If $S$ has no tangent of infinite order and $\alpha=\alpha(n)=[(n+3) / 2]$, then the Fourier transform of the measure $\mu_{\alpha}$ with $d \mu_{\alpha}=\kappa^{\alpha} w d S$ satisfies the estimate

$$
\left|\hat{\mu}_{\alpha}(\theta)\right| \leq C(n, w, S)|\theta|^{-n / 2} \quad \forall \theta \in \mathbf{R}^{n+1} .
$$

PROOF. We shall represent the surface $S$ in a neighborhood of each point $p$ in $\operatorname{supp}(w)$ as the graph of a convex function defined on the tangent plane $T_{p}$ at $p$. Introducing polar coordinates in $T_{p}$ we shall reduce matters to the estimation of a one-dimensional oscillatory integral of the type studied in the previous section. A similar approach has been previously used by several authors to estimate these Fourier transforms [He, Sv, R].

Since $S$ is strictly convex, for every vector $\Theta$ in the unit sphere $\Sigma_{n}$ in $\mathbf{R}^{n+1}$ there exists at most one point $X(\Theta)$ on $S$ with interior normal $\Theta$, i.e. $(x-X(\Theta)) \cdot \Theta \geq 0$ for every $x \in S$. Let $\Sigma_{n}^{\prime}$ be the compact subset of $\Sigma_{n}$ consisting of those $\Theta$ for 
which $X(\Theta)$ exists and belongs to the support of $w$. Since the function $\kappa^{\alpha} w$ is smooth, by a well-known argument (see for instance $[\mathbf{L}]$ ) the contribution to

$$
\hat{\mu}(\lambda \Theta)=\int_{S} e^{i \lambda \Theta \cdot x} \kappa^{\alpha}(x) w(x) d S(x) \quad \forall \lambda \in \mathbf{R}
$$

coming from portions of $S$ outside a small neighborhood of the points $X(\Theta)$ and $X(-\Theta)$ is a rapidly decreasing function of $\lambda$ as $|\lambda| \rightarrow \infty$. Thus it is enough to examine the contribution to (5.1) coming from a small neighborhood $N(\Theta)$ of $X(\Theta)$, whose radius can be chosen to be independent of $\Theta$, and it suffices to prove the estimate

$$
\left|\int_{S} e^{i \lambda \Theta \cdot x} \kappa^{\alpha}(x) w_{1}(x) d S(x)\right| \leq c(n, w, S)|\lambda|^{-n / 2} \quad \forall \lambda \in \mathbf{R}
$$

for $w_{1} \in C_{c}^{\infty}(N(\Theta))$. Now consider a rotation and translation of $\mathbf{R}^{n+1}$ so that the point $X(\Theta)$ is moved to the origin, and the tangent plane of $S$ at $X(\Theta)$ becomes the hyperplane $x_{n+1}=0$. Then in a ball $B_{\delta}$ centered at the origin, of radius $\delta$ that can be chosen independent of $\Theta$, the surface $S$ can be given as the graph of a smooth convex function $f_{\Theta}: B_{\delta} \rightarrow \mathbf{R}$ such that $f_{\Theta}(0)=0, f_{\Theta}^{\prime}(0)=0$, and further the map $\Theta \rightarrow f_{\Theta}$ is continuous from $\Sigma_{n}^{\prime}$ to $C^{m}\left(B_{\delta}\right)$ for every $m \in \mathbf{N}$. Henceforth we shall write $f$ instead of $f_{\Theta}$, for the sake of brevity, unless we want to stress explicitly the dependence of $f$ on $\Theta$. Then estimate (5.2) is equivalent to the estimate

$$
\left|\int_{\mathbf{R}^{n}} e^{i \lambda f(x)}\left(\operatorname{det} f^{\prime \prime}(x)\right)^{\alpha} w_{2}(x) d x\right| \leq c(n, w, S)|\lambda|^{-n / 2} \quad \forall \lambda \in \mathbf{R},
$$

where $w_{2}(\cdot)=w_{2}(\cdot, \Theta) \in C_{c}^{\infty}\left(B_{\delta}\right)$ and the map $\Theta \rightarrow w_{2}(\cdot, \Theta)$ is continuous from $\Sigma_{n}^{\prime}$ into $C^{m}\left(B_{\delta}\right)$ for every $m \in \mathbf{N}$. Next we introduce polar coordinates $t, \xi$ in $\mathbf{R}^{n}$ and we put

$$
\phi(t, \xi, \Theta)=f_{\Theta}(t) \quad \forall t \geq 0, \forall \xi \in \Sigma_{n-1} .
$$

In terms of these new coordinates the integral in (5.3) becomes

$$
\int_{\Sigma_{n-1}} d \xi \int_{0}^{\delta} \exp (i \lambda \phi(t, \xi, \Theta)) \psi^{\alpha}(t, \xi, \Theta) u(t, \xi, \Theta) t^{n-1} d t,
$$

where $\psi(t, \xi, \Theta)=\operatorname{det}\left(f_{\Theta}^{\prime \prime}(x)\right)$ and $u(t, \xi, \Theta)=w_{2}(t, \xi, \Theta)$. For every $m \in \mathbf{N}$ the maps

$$
(\xi, \Theta) \rightarrow \phi(\cdot, \xi, \Theta), \quad(\xi, \Theta) \rightarrow \psi(\cdot, \xi, \Theta), \quad(\xi, \Theta) \rightarrow u(\cdot, \xi, \Theta)
$$

are continuous from $\Sigma_{n-1} \times \Sigma_{n}^{\prime}$ into $C^{m}([0, \delta])$. Hence their image is a compact subset of $C^{m}([0, \delta])$. Moreover for every $(\xi, \Theta) \in \Sigma_{n-1} \times \Sigma_{n}^{\prime}$ the function $t \rightarrow$ $u(t, \xi, \Theta)$ vanishes in a neighborhood of $\delta$; moreover, the function $t \rightarrow \phi(t, \xi, \Theta)$ is convex, satisfies $\phi(0, \xi, \Theta)=\partial_{t} \phi(0, \xi, \Theta)=0$ and, since $S$ has no tangent of infinite order, there exist an integer $q \geq 2$ and a positive constant $\varepsilon$, both independent of $(\xi, \Theta)$, such that

$$
\max \left\{\left|\partial_{t}^{i} \phi(0, \xi, \Theta)\right|: 2 \leq i \leq q\right\} \geq \varepsilon .
$$

We now need a geometric lemma.

LEMMA 5.2. There exists a constant $c_{0}$, independent of $(\xi, \Theta)$, such that

$$
0 \leq \psi(t, \xi, \Theta) \leq c_{0} \partial_{t}^{2} \phi(t, \xi, \Theta) \quad \forall t \in[0, \delta] .
$$


Proof. Let $f$ be a convex $C^{2}$ function on an open subset $\Omega$ of $\mathbf{R}^{n}$. Then for every vector $\xi$ in $\Sigma_{n-1}$,

$$
\operatorname{det} f^{\prime \prime}(y) \leq\left\|f^{\prime \prime}(y)\right\|^{n-1}\left(f^{\prime \prime}(y) \xi \cdot \xi\right)
$$

for all $y \in \Omega$. Indeed let $\xi_{1}, \ldots, \xi_{n}$ be an orthonormal basis of eigenvectors of $f^{\prime \prime}(y)$ and denote by $C_{1}, \ldots, C_{n}$ the corresponding eigenvalues. Let $\xi=\sum \mu_{i} \xi_{i}$, $\sum \mu_{i}^{2}=1$, be a unit vector in $\mathbf{R}^{n}$. Then, since $0 \leq C_{i} \leq\left\|f^{\prime \prime}(y)\right\|$, we have

$$
\begin{aligned}
\operatorname{det} f^{\prime \prime}(y) & =\prod_{i=1}^{n} C_{i} \leq\left\|f^{\prime \prime}(y)\right\|^{n-1} \prod_{i=1}^{n} C_{i}^{\mu_{i}^{2}} \\
& \leq\left\|f^{\prime \prime}(y)\right\|^{n-1} \sum_{i=1}^{n} \mu_{i}^{2} C_{i}=\left\|f^{\prime \prime}(y)\right\|^{n-1}\left(f^{\prime \prime}(y) \xi \cdot \xi\right)
\end{aligned}
$$

by the inequality between the geometric and arithmetic means. By applying inequality (5.4) to the function $f_{\Theta}$ we obtain the desired conclusion.

PROOF OF THEOREM 5.1 (CONTINUED). Now, if $n$ is even, say $n=2 k$, then $\alpha(n)=k+1$ and Proposition 4.4 yields

$$
\begin{gathered}
\left|\int_{0}^{\delta} \exp (i \lambda \phi(t, \xi, \Theta)) \psi^{\alpha(n)}(t, \xi, \Theta) u(t, \xi, \Theta) u(t, \xi, \Theta) t^{n-1} d t\right| \\
\leq c\left(n, \delta^{\prime} \Sigma\right)|\lambda|^{-n / 2} \quad \forall \lambda \in \mathbf{R},
\end{gathered}
$$

from which estimate (5.3) follows easily. If $n$ is odd we increase the dimension to $n+1$ by considering the function $F$ defined on a ball of center 0 and radius $\delta$ in $\mathbf{R}^{n+1}$ by

$$
F\left(x_{1}, \ldots, x_{n+1}\right)=f\left(x_{1}, \ldots, x_{n}\right)+\frac{1}{2} x_{n+1}^{2} .
$$

Given a point $x \in \mathbf{R}^{n+1}$ we shall write $x=\left(x^{\prime}, x_{n+1}\right)$ where $x^{\prime}=\left(x_{1}, \ldots, x_{n}\right)$. Then $\operatorname{det} F^{\prime \prime}(x)=\operatorname{det} f^{\prime \prime}\left(x^{\prime}\right)$. Let $w_{3}$ in $C_{c}^{\infty}(-\delta, \delta)$ be a function such that $w_{3}(0)=1$. Then, since $n+1$ is even, $\alpha(n+1)=\alpha(n)$, and the previous argument yields

$$
\left|\int_{\mathbf{R}^{n+1}} \exp (i \lambda F(x))\left(\operatorname{det} F^{\prime \prime}(x)\right)^{\alpha(n)} w_{2}\left(x^{\prime}\right) w_{3}\left(x_{n+1}\right) d x\right| \leq c|\lambda|^{-(n+1) / 2} .
$$

On the other hand the integral in (5.5) factors into the product of

$$
\int_{\mathbf{R}^{n+1}} \exp \left(i \lambda f\left(x^{\prime}\right)\right)\left(\operatorname{det} f^{\prime \prime}\left(x^{\prime}\right)\right)^{\alpha(n)} w_{2}\left(x^{\prime}\right) d x^{\prime}
$$

and

$$
\left(\int_{\mathbf{R}} \exp \left(\frac{i}{2} \lambda x_{n+1}^{2}\right) w_{3}\left(x_{n+1}\right) d x_{n+1}\right) .
$$

Since by [E, Chapter II],

$$
\int_{\mathbf{R}} \exp \left(\frac{i}{2} \lambda x_{n+1}^{2}\right) w_{3}\left(x_{n+1}\right) d x_{n+1} \sim \operatorname{sgn}(\lambda)\left(\frac{|\lambda|}{2 \pi i}\right)^{-1 / 2} \quad \text { as }|\lambda| \rightarrow \infty,
$$

estimate (5.3) holds also for $n$ odd. 


\section{REFERENCES}

[CM] M. Cowling and G. Mauceri, Inequalities for some maximal functions. II, Trans. Amer. Math. Soc. 296 (1986), 341-365.

[E] A. Erdélyi, Asymptotic expansions, Dover, 1970.

[G] A. Greenleaf, Principal curvature and harmonic analysis, Indiana Math. J. 30 (1982), 519-537.

[He] C. Herz, Fourier transforms related to convex sets, Ann. of Math. (2) 75 (1961), 81-92.

[H] L. Hörmander, The analysis of linear partial differential operators. I, Springer-Verlag, Berlin, Heidelberg, New York, and Tokyo, 1982.

[L] W. Littman, Fourier transforms of surface carried measures and differentiability of surface averages, Bull. Amer. Math. Soc. 69 (1963), 766-770.

[R] B. Randol, On the asymptotic behavior of the Fourier transform of the indicator function of a convex set, Trans. Amer. Math. Soc. 139 (1969), 279-285.

[S] E. M. Stein, Maximal functions: spherical means, Proc. Nat. Acad. Sci. U.S.A. 73 (1976), 2174-2175.

[SS] C. D. Sogge and E. M. Stein, Averages of functions over hypersurfaces in $\mathbf{R}^{n}$, Invent. Math. 82 (1985), 543-556.

[SW] E. M. Stein and S. Wainger, Problems in harmonic analysis related to curvature, Bull. Amer. Math. Soc. 84 (1978), 1239-1295.

[Sv] I. Svensson, Estimates for the Fourier transform of the characteristic function of a convex set, Ark. Mat. 9 (1971), 11-22.

School of Mathematics, University of New South Wales, Sydney, New South Wales, Australia

Department of Mathematics, University of Genova, Genova, italy (Current address of Giancarlo Mauceri)

Current address (Michael Cowling): Department of Mathematics, University of Rome-Tor Vergata, Rome, Italy 